\title{
A comprehensive usability model for evaluating smartphone apps
}

\begin{abstract}
This paper proposes a model for usability evaluation in view of smartphone characteristic, user interface design, and context of use approach. A set of mobile related bibliographic has been reviewed. Consequently, 4 contexts, 12 usability features, 31 usability criteria have been identified. Later, the reviewed items are classified according to the identified context, usability features, and usability attributes. The model is then validated using expert evaluation in an online survey. The survey result is compared with a simulated response to verify its accuracy. It is found out that both resulted with at least $90 \%$ of the field experts agreed with the operational definition of the four usability context, the identified 12 usability features and 31 corresponding usability criteria. Relevant feedbacks received from the survey were adopted into the model. While previous studies evaluate apps based on either by traditional computing design elements, ergonomics or HCI perspectives, this paper address usability of apps by incorporating measurement from previous studies and user interface elements of apps adopted in the industry while at the same time, acknowledging the different set of evaluator skill.
\end{abstract}

Keyword: Mobile usability; Smartphone app; Usability checklist; Usability model 